\title{
ON CSISZÁR AND TSALLIS TYPE $f$-DIVERGENCES INDUCED BY SUPERQUADRATIC AND CONVEX FUNCTIONS
}

\author{
PaweŁ A. Kluza And MareK Niezgoda
}

\begin{abstract}
In this paper, Csiszár and Tsallis $f$-divergences are studied for superquadratic and convex functions. Some comparison theorems for two divergences are provided. The obtained results, when used for nonnegative superquadratic functions, give some refinements of the original inequalities corresponding to nonnegative convex functions. Some majorization assumptions for the involved matrix are simplified from column stochasticity to entrywise-nonnegativity.
\end{abstract}

Mathematics subject classification (2010): 26D15, 15B51, 94A17.

Keywords and phrases: convex function, superquadratic function, Csiszár $f$-divergence, Tsallis $f_{u}$ divergence, column stochastic matrix.

\section{REFERENCES}

[1] S. Abramovich, G. Jameson And G. Sinnamon, Inequalities for averages of convex and superquadratic functions, J. Inequal. Pure Appl. Math. 5 (2004), Art. 91.

[2] S. Abramovich, G. Jameson and G. Sinnamon, Refining Jensen's inequality, Bull. Math. Soc. Sci. Math. Roumanie (N. S.), 47, 95 (2004), 3-14.

[3] S. Abramovich, S. IVelić And J. E. PeČARIĆ, Improvement of Jensen-Steffensen's inequality for superquadratic functions, Banach J. Math. Anal. 4 (2010), 159-169.

[4] J. C. BAEZ, Rényi entropy and free energy, J. Stat. Mech. Theory E. (2011), arXiv:1102 2098.

[5] J. C. BAez AND B. S. Pollard, Relative entropy in biological systems, Entropy 18, 246 (2016), doi: $10.3390 / \mathrm{e} 18020046$.

[6] G. E. Crooks, On measures of entropy and information, Tech. Note 009, v0.5, (2016), 2016-08-16, http://threeplusone.com/info.

[7] G. E. CROoKS AND D. A. SivaK, Measures of trajectory ensemble disparsity in nonequilibrium statistical dynamics, J. Stat. Mech. Theory E., (2011) P06003.

[8] I. CSISZÁR, Information-type measures of differences of probability distributions and indirect observations, Studia Sci. Math. Hung. 2 (1967), 299-318.

[9] I. CSISZÁR AND J. KÖRnER, Information Theory: Coding Theorems for Discrete Memory-less Systems, Academic Press, New York, 1981.

[10] S. S. DRAGOMIR, Upper and lower bounds for Csiszár $f$-divergence in terms of the Kullback-Leibler distance and applications, in Inequalities for the Csiszár $f$-divergence in Information Theory, ed. S. S. Dragomir, 2000, http://rgmia.vu.edu.au/monographs/csiszar.htm.

[11] P. A. KLuZA AND M. NiezGODA, Inequalities for relative operator entropies, Electron. J. Linear Algebra 27 (2014), 851-864.

[12] P. KluZa AND M. NiezGoda, Generalizations of Crooks and Lin's results on Jeffreys-Csiszár and Jensen-Csiszár f-divergences, Physica A, 463 (2016), 383-393.

[13] J. Lin, Divergence measures based on the Shannon entropy, IEEE Trans. Inf. Th. 37, 1 (1991), 145151.

[14] F.-C. Mitroi-Symeonidis And N. Minculete, On the Jensen functional and superquadraticity, Aequationes Math. 90, 4 (2016), 705-718.

[15] F.-C. Mitroi-Symeonidis And N. Minculete, On the Jensen functional and strong convexity, Bull. Malays. Math. Sci. Soc., 2016, doi:10.1007/s40840-015-0293- z. 
[16] M. NiEZgoda, Shannon like inequalities for $f$-connections of positive linear maps and positive operators, Linear Algebra Appl. 481 (2015), 186-201.

[17] M. NiEZGODA, Vector joint majorization and generalization of Csiszár-Körner's inequality for $f$ divergence, Discrete Appl. Math. 198 (2016), 195-205.

[18] M. NiezGoda, Inequalities for $H$-invex functions with applications for uniformly convex and superquadratic functions, Filomat 31, 15 (2017), 4781-4794.

[19] C. TS AlLis, Possible generalization of Bolzmann-Gibbs statistics, J. Stat. Phys. 52 (1988,) 479-487.

[20] S. Sherman, On a theorem of Hardy, Littlewood, Pólya, and Blackwell, Proc. Nat. Acad. Sci. USA, 37 (1957), 826-831. 